\title{
Fisetin inhibits liver cancer growth in a mouse model: Relation to dopamine receptor
}

\author{
XIANG-FENG LIU, HAI-JIAO LONG, XIONG-YING MIAO, GUO-LI LIU and HONG-LIANG YAO \\ Department of General Surgery, The Second Xiangya Hospital of Central South University, \\ Changsha, Hunan 421000, P.R. China
}

Received November 17, 2016; Accepted May 3, 2017

DOI: $10.3892 / o r .2017 .5676$

\begin{abstract}
Fisetin (3,3',4',7-tetrahydroxyflavone), a natural abundant flavonoid, is produced in different vegetables and fruits. Fisetin has been reported to relate to various positive biological effects, including anti-proliferative, anticancer, anti-oxidative and neuroprotective effects. Dopamine receptors (DRs) belonging to $\mathrm{G}$ protein-coupled receptor family, are known as the target of $\sim 50 \%$ of all modern medicinal drugs. DRs consist of various proteins, functioning as transduction of intracellular signals for extracellular stimuli. We found that fisetin performed as DR2 agonist to suppress liver cancer cells proliferation, migration and invasion. Caspase-3 signaling was activated to induce apoptosis for fisetin administration. Furthermore, TGF- $\beta 1$ was also inhibited in fisetin-treated liver cancer cells, reducing epithelial-mesenchymal transition (EMT). Additionally, fisetin downregulated VEGFR1, p-ERK1/2, p38 and pJNK, ameliorating liver cancer progression. In vivo, the orthotopically implanted tumors from mice were inhibited by fisetin adminisatration accompanied by prolonged survival rate and higher levels of dopamine. Together, the results indicated a novel therapeutic strategy to suppress liver cancer progression associated with DR2 regulation, indicating that dopamine might be of importance in liver cancer progression.
\end{abstract}

\section{Introduction}

Hepatocellular carcinoma (HCC) is a well known primary liver cancer with very poor prognosis (1). According to a previous report, HCC is the sixth most common cancer (2). Furthermore, it is the third leading reason for cancer mortality across the world (3). Each year, $>600,000$ deaths are reported due to its difficult diagnosis and ineffective treatments $(4,5)$. The incidence rate of liver cancer is increasing among both

Correspondence to: Dr H.L. Yao, Department of General Surgery, The Second Xiangya Hospital of Central South University, 139 Renmin Road, Changsha, Hunan 421000, P.R. China

E-mail: yhlhunan421000@qq.com; or yao@126.com

Key words: fisetin, dopamine receptors, liver cancer, apoptosis, TGF- $\beta 1$ male and female, and the number of people infected with HCC has doubled (6,7). Until now, chemoprevention has been reported as the best treatment to prevent liver cancer progression and development (8).

Presently, accumulating evidence suggests that a variety of natural compounds isolated from plants are safe and effective in many diseases, including cancer, such as liver cancer, breast cancer and lung cancer (9). Thus, we attempted to illustrate the role of fisetin (3,3',4',7-tetrahydroxyflavone) (Fig. 1A) in HCC prevention or treatment. Fisetin is found in various vegetables and fruits, including onions, cucumber, apples, grapes, and strawberries (10). In addition, fisetin is suggested to possess anti-oxidant, anti-microbio, anti-inflammation and even anticancer properties in a variety of animal models as well as cell cultures (11). Additionally, apoptosis in cancer cells, such as cervical and breast, could be induced by fisetin, contributing to inhibition of cancer progression (12). Therefore, we hypothesized that fisetin could be of potential value in human liver cancer inhibition.

Dopamine (DA) is essential in sodium balance and blood pressure modulation, which directly functions on lung, renal, brain, and vascular bed ion transport (13). Five DA receptors have been reported, indlucing DR1, DR2, DR3, DR4 an DR5 $(14,15)$. DR1 and DR5 are regarded as members of D1-like family, and DR2, DR3 and DR4 belong to D2-like family (16). DA has been reported to have anticancer activities, such as lung cancer, gastric cancer and cervical cancer, mainly via DR2 regulation (17). According to the possible role of DR2 in tumor suppression, it was also included in our study to explore whether it was related to liver cancer progression. Thus, DR2 agonist in our study was used to explore how DRs perform in liver cancer in vitro. Fisetin was used combined with DR2 agonist to investigate underlying molecular mechanism related to $\mathrm{HCC}$ progression.

\section{Materials and methods}

Cells and culture. Human liver caner cell lines, HCC-LM3 and SMMC-7721, were purchased from American Type Culture Collection (ATCC, USA). Human liver cancer cells of HepG2 were purchased from KeyGen Biotech (Nanjing, China). SMMC-7721 and HepG2 cells were cultured in DMEM (Gibco, USA) supplemented with $10 \% \mathrm{FBS}, 100 \mathrm{U} / \mathrm{ml}$ penicillin, and $100 \mu \mathrm{g} / \mathrm{ml}$ streptomycin. Human HCC-LM3 cells were 
routinely cultured in RPMI-1640 medium (Gibco), containing $10 \%$ fetal bovine serum (FBS) (Gibco), $1 \%$ penicillin/streptomycin. MRC-5 was cultured in DMEM (Gibco) supplemented with $10 \%$ FBS, $100 \mathrm{U} / \mathrm{ml}$ penicillin, and $100 \mu \mathrm{g} / \mathrm{ml}$ streptomycin. All cells were cultured in a humidified atmosphere with $5 \% \mathrm{CO}_{2}$ and $95 \%$ humidity at $37^{\circ} \mathrm{C}$ in an incubator. All cells were cultured in a humidified atmosphere with $5 \% \mathrm{CO}_{2}$ and $95 \%$ humidity at $37^{\circ} \mathrm{C}$ in an incubator. Fisetin ( $>98 \%$ purity), used for the treatment of lung cancer, was purchased from Dayang Chem (Hangzhou, China), dissolved in DMSO and stored at $-20^{\circ} \mathrm{C}$, and then diluted in medium for experiments. The final DMSO concentration in our study was $<0.1 \%(\mathrm{v} / \mathrm{v})$ in every treatment, and bromocriptine used was purchased from Sigma-Aldrich (St. Louis, MO, USA).

3-(4, 5-dimethylthiazol-2-yl)-2, 5-diphenyl-2H-tetrazolium bromide (MTT) assays. Cells of HepG2 (5x103), HCC-LM3 and SMMC-7721 were seeded into a 96-well plate (Corning, USA) per well. Bromocriptine (from 0 to $40 \mu \mathrm{M}$ ), fisetin ranging from 0-40 $\mu \mathrm{M}$, was added to the medium. The cells were then incubated at $37^{\circ} \mathrm{C}$ for $24 \mathrm{~h}$, and the cell viability was detected by the colorimetric MTT assay at $570 \mathrm{~nm}$.

Cell migration and invasion analysis for liver cancer cells. The liver cancer cells were seeded into the upper chamber of a Transwell insert pre-coated with $5 \mu \mathrm{g} / \mathrm{ml}$ fibronectin for migration or a $\mathrm{BD}^{\mathrm{TM}}$ Matrigel invasion chamber for invasion. Medium with $10 \%$ serum was put in the lower chamber as a chemo-attractant, and cells were then incubated for $4 \mathrm{~h}$. Non-migratory cells were removed from the upper chamber with a cotton bud. The cells on the lower insert surface were stained with Diff-Quick. Cells were counted as the number of cells observed in three randomly different microscope fields of three independent inserts.

Colony-forming analysis of liver cancer cells. To explore the liver cancer cell proliferation, $60 \%$ liver cancer cells of HCC-LM3 and HepG2 were treated with bromocriptine and fisetin or the two drugs in combination for $24 \mathrm{~h}$ in growth medium. The cells were then harvested in a separate tube after incubation. Then, 500 cells/well were cultured in 6-well plate (Corning) separately with complete growth medium and grown for 2 weeks. The medium was replaced after 7 days. For incubation of 14 days, the cells were washed with phosphatebuffered saline (PBS) (Hyclone, USA). Then, cold methanol was used to fix cells for $10 \mathrm{~min}$. Cells were stained with $0.5 \%$ crystal violet solution (Chemcatch, USA) in 25\% methanol at room temperature. The cells were washed with water three times and air-dried for calculating through an inverted microscope.

DA analysis. ELISA kits for DA determination in serum and tumor tissue samples from mice were purchased from Nordhorn (Germany). DA detection was performed following the manufacturer's instructions.

Wesertn blot analysis. After treatments under different conditions, the cells were harvested and the medium was removed. Then the cells were washed with chilled PBS three times and lysed in ice-cold lysis buffer with fresh protease inhibitor cocktail. The cell lysates were centrifuged at $12,000 \mathrm{~g}$ for $20 \mathrm{~min}$ at $4^{\circ} \mathrm{C}$ to collect the supernatant. BSA protein assay kit was used to detect the protein concentrations following the manufacturer's instructions (Thermo, USA). Protein extracts (40-ng) were separated by $10 \%$ SDS-PAGE and were then transferred to polyvinylidene fluoride membrane (Millipore, USA). The PVDF with proteins were blocked with 5\% skim fat dry milk in $0.1 \%$ Tween-20 in Tris-buffered saline (TBS) for $2 \mathrm{~h}$ to block the non-specific sites on blots. The primary antibodies dissolved in blocking buffer were used to detect the target protein blots at $4^{\circ} \mathrm{C}$ overnight for incubation. The bands on PVDF were covered by chemiluminescence with Pierce ECL Western Blotting Substrate reagents (Thermo Scientific, IL, USA). The primary antibodies used in our study were: rabbit anti-GAPDH, caspase-3, PARP, TGF- $\beta 1$, VEGFR1, ERK1/2, p-ERK1/2, p38, pJNK, E-cadherin, N-cadherin, and vimentin.

Flow cytometry assays. The Annexin V-FITC/propidium iodide (PI) apoptosis detection kit (KeyGen) was used to determine apoptotic cells. Liver cancer cells after different treatments were harvested and washed with chilled PBS, then incubated in a darkroom for Annexin V-FITC and PI for $15 \mathrm{~min}$. Subsequently, the cells were analyzed by flow cytometry (BD Biosciences, USA).

The hepatoma model. Sixty, 6-week old male, athymic BALB/C nude mice were purchased from the Animal Center of Nanjing Medical University (Nanjing, China) and cultured in a $25 \pm 2^{\circ} \mathrm{C}$ temperature and $50 \pm 10 \%$ humidity-controlled environment with a standard 12-h light and dark cycle with food and water in cages under germ-free conditions. All processes were in line with the Institutional Animal Care and Use Committee of the second Xiangya Hospital of Central South University. Briefly, athymic BALB/C nude mice were implanted orthotopically with liver cancer HCC-LM3 cells ( $3 \times 10^{6}$ cells). All tumor-bearing mice were divided into 4 groups randomly: i) control; ii) Fis $(20 \mathrm{mg} /$ $\mathrm{kg})$; iii) Fis $(40 \mathrm{mg} / \mathrm{kg})$; iv) Fis $(80 \mathrm{mg} / \mathrm{kg})$ daily through i.p. After 7 weeks of treatment, the mice were sacrificed for the following experiments. The blood from mouse was obtained through the eyeball, and then was centrifuged at $15,000 \mathrm{~g}$ for 15 min. Fisetin was dissolved in DMSO and diluted in distilled water for further use.

Immunohistochemical assays. The tissue in each group were fixed with $10 \%$ buffered formalin, embedded in paraffin and sliced into $4-5-\mu \mathrm{m}$ thick sections. Tumor tissues also were subjected to immunohistochemical (IHC) staining for the analysis of VEGFR1 and Ki-67 expression. The immunfluorescent analysis was carried out as described previously (18). The cells or tissue samples were incubated with TGF- $\beta 1$, as the primary antibody, overnight at $4^{\circ} \mathrm{C}$. The slides were then washed with cold PBS and incubated with anti-rabbit secondary antibody (KeyGen). The histological examination was performed following the standard procedures described previously (19).

Statistical analysis. Data were expressed as mean \pm SEM. Statistical analyses were carried out with GraphPad PRISM 
A<smiles>O=C1CC(c2ccc(O)c(O)c2)Oc2cc(O)ccc21</smiles>

C

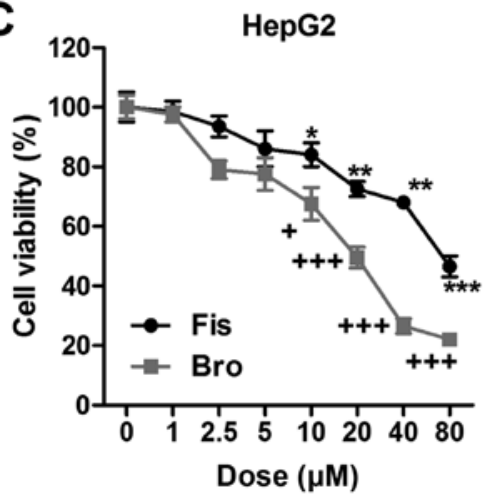

B

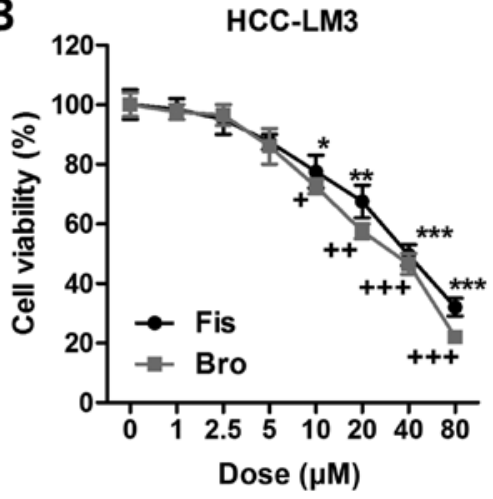

D

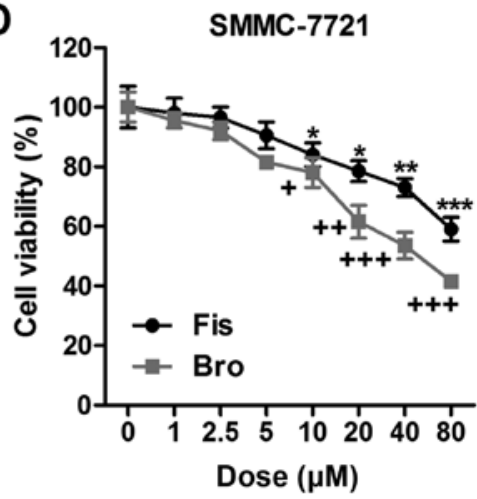

Figure 1. Fisetin-suppresses liver cancer cells proliferation related to death receptor 2 (DR2). (A) The chemical structure of fisetin is shown. Liver cancer cells of (B) HCC-LM3, (C) HepG2, and (D) SMMC-7721 were treated with different concentrations of fisetin $(0,1,2.5,5,10,20,40$ and $80 \mu$ M) and bromocriptine $(0,1,2.5,5,10,20,40$ and $80 \mu \mathrm{M})$ for $24 \mathrm{~h}$, then the cell proliferation and toxicity was determined via MTT analysis. Data are presented as mean \pm SEM. ${ }^{*} \mathrm{P}<0.05,{ }^{* *} \mathrm{P}<0.01$ and ${ }^{* * *} \mathrm{P}<0.001$ versus Con group with Fis treatment; ${ }^{+} \mathrm{P}<0.05,{ }^{++} \mathrm{P}<0.01$ and ${ }^{+++} \mathrm{P}<0.001$ versus Con group with Bro treatment. Fis, fisetin; Bro, bromocriptine.

(version 6.0; Graph Pad Software). A P-value <0.05 was considered statistically significant.

\section{Results}

Fisetin-suppresses liver cancer cell proliferation related to death receptor 2 (DR2). In order to explore whether fisetin could perform as DR2 agonist showing important role in regulating liver cancer cell prolifreration, the cell viability of liver cancer cells of HCC-LM3, HepG2 and SMMC-7721 was calculated. As shown in Fig. 1B, fisetin at different concentrations $(0,1$, $2.5,5,10,20,40$ and $80 \mu \mathrm{M})$ was administered to HCC-LM3 cells. Fisetin showed significantly inhibitory role in HCC-LM3 proliferation $>10 \mu \mathrm{M}$. Similarly, the death receptor 2 agonist of bromocriptine markedly suppressed HCC-LM3 cell proliferation in a dose-dependent manner. Consistently, in HepG2 cells, fisetin also displayed suppressive effects on cell proliferation, which was in line with the role of bromocriptine in HepG2 regulation (Fig. 1C). Fisetin also exhibited inhibitory role in SMMC-7721 cells. Of note, bromocriptine, as DR2 agonist, reduced the liver cancer cell viability (Fig. 1D). Taken together, the results above indicated that fisetin shows similar role with bromocriptine, reducing liver cancer cell lines proliferation, which might be an essential therapeutic strategy for liver cancer treatment and linked with the DR2 signal.

Effects of fisetin and bromocriptine on liver cancer cell proliferation, migration and invasion. In this regard, we investigated the effects of fisetin and bromocriptine treatment on liver cancer cell proliferation, migration and invasion. Whether the treatment of fisetin and bromocriptine influenced the clonogenic growth of HCC-LM3 and HepG2, colonyforming analysis was also carried out. In Fig. 2A and B, the invasion of liver cancer cells were apparently reduced due to fisetin and bromocriptine treatment in HCC-LM3 cells. Notably, fisetin and bromocriptine co-treatment could further downregulate the number of invaded cells, suggesting that fisetin might share similar molecular mechanism with bromocriptine to regulate liver cancer progression. Also, in HepG2 cells, fisetin and bromocriptine reduced cell invasion, which was further enhanced for fisetin and bromocriptine combination (Fig. 2A and C). Next, fisetin and bromocriptine obviously reduced HCC-LM3 cell migration. Also, intensively suppressive role of fisetin and bromocriptine co-treatment in cell migration inhibition was observed (Fig. 2D and E). In addition, fisetin and bromocriptine could considerably downregulate HepG2 cell migration, which was promoted for fisetin and bromocriptine in combination (Fig. 2D and F). Finally, colony formation assays showed that fisetin and bromocriptine monotherapy significantly reduced the colony number of cancer cells compared to the control ones. Notably, combination of fisetin and bromocriptine markedly decreased the clonogenic growth of lung cancer cells of HCC-LM3 and HepG2 (Fig. 2G-I). The results illustrate the capability of fisetin and bromocriptine to suppress liver cancer cell invasion migration, and proliferation is appar- 
A

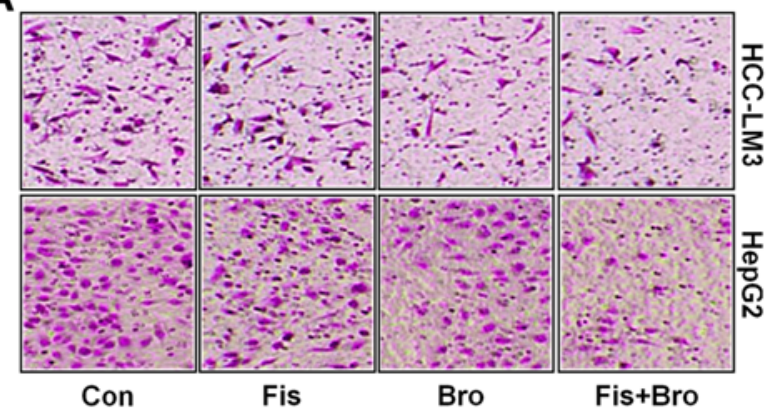

D

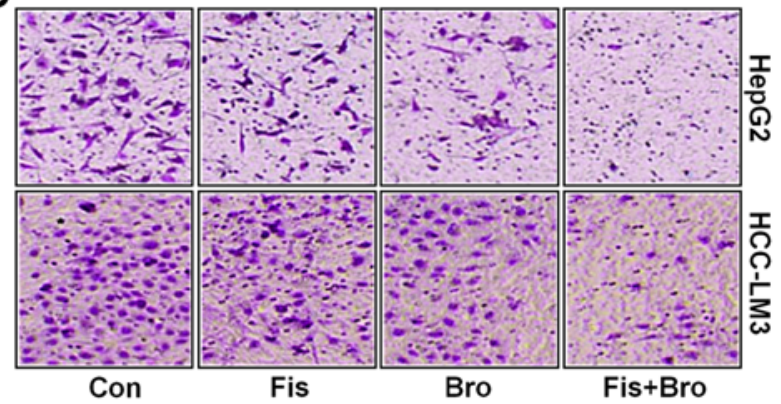

G

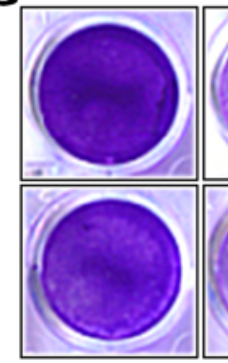

Con

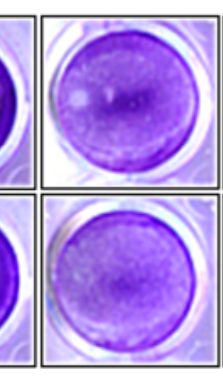

Fis

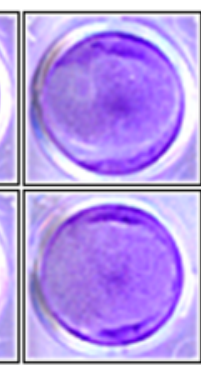

Bro

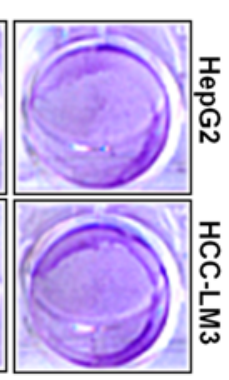

Fis+Bro
B

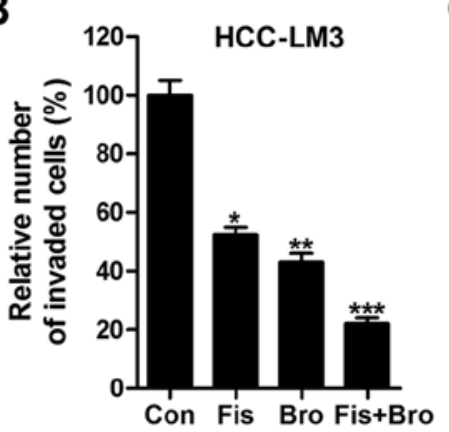

E

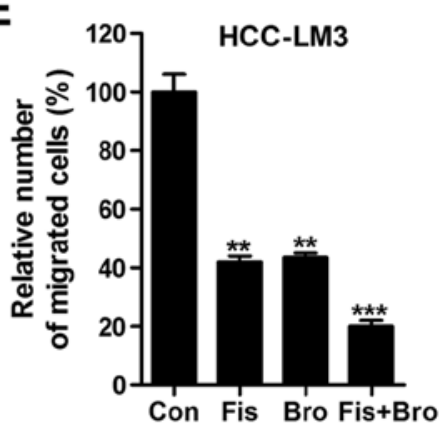

H

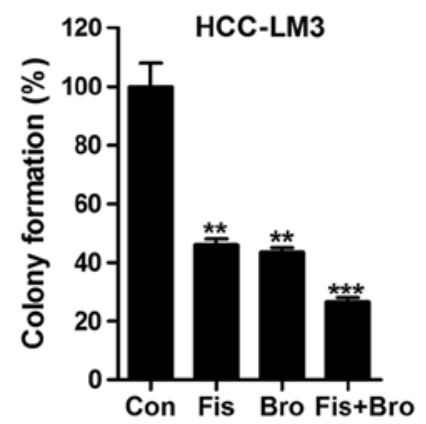

C

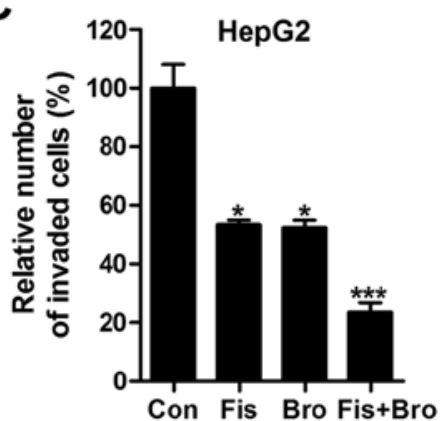

$\mathbf{F}$

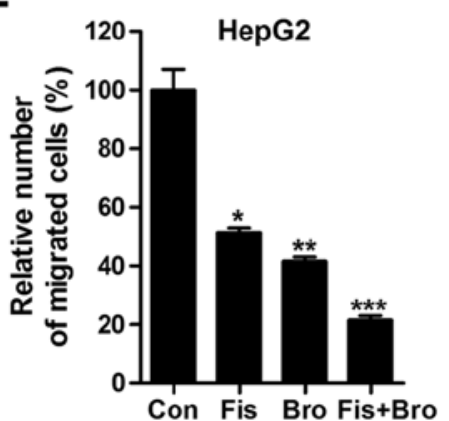

(

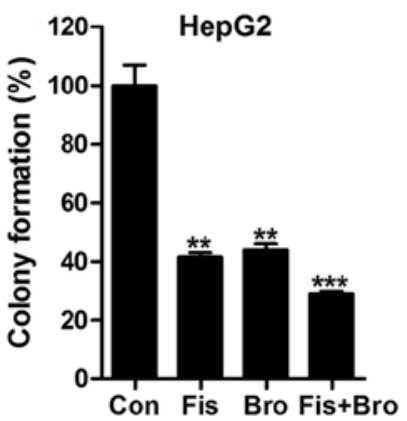

Figure 2. Effects of fisetin and bromocriptine on liver cancer cell proliferation, migration and invasion. (A) The reprentative images of invaded liver cancer cells of MCC-LM3 and HepG2 after fisetin $(40 \mu \mathrm{M})$ and bromocriptine $(40 \mu \mathrm{M})$ and the two-drug combination treatments. (B) The quantification of HCC-LM3 invasion is displayed. (C) The quantification of HepG2 invasion was calculated. (D) The reprentative images of migrated liver cancer cells of MCC-LM3 and HepG2 after fisetin $(40 \mu \mathrm{M})$ and bromocriptine $(40 \mu \mathrm{M})$ and the two-drug combination treatments are shown. (E) The quantification of HCC-LM3 migration. (F) The quantification of HepG2 migration was calculated. (G) The colony-forming ability of liver cancer cells after treatment with fisetin $(40 \mu \mathrm{M})$ and/or bromocriptine $(40 \mu \mathrm{M})$ was detected. (H) The clonogenic growth of MCC-LM3 was quantified. (I) The quantification of HepG2 cell clonogenic growth is shown. The experiments were repeted at least three times. Data are presented as mean $\pm \mathrm{SEM}$. ${ }^{*} \mathrm{P}<0.05,{ }^{* *} \mathrm{P}<0.01$ and ${ }^{* * * *} \mathrm{P}<0.001$ versus Con group without any treatment.

ently stronger than the effect of fisetin and bromocriptine separately performed in the present experiments, and fisetin, at least partly, could perform as bromocriptine in controlling liver cancer progression.

Fisetin and bromocriptine induce apoptosis in liver cancer cells via caspase-3 activation. Apoptosis induction is known as a key mechanism, contributing to cell death, which is widely used to explore and find new therapeutic strategy (20). In this study, the flow cytometric results exhibited that the fisetin and bromocriptine significantly upregulated the apoptotic liver cancer cells HCC-LM3 and HepG2. Interestingly, fisetin and bromocriptine combination caused an obvious upregulation of apoptotic cells compared to the control ones (Fig. 3A-C). Next, the caspase activation of cancer cells after fisetin and bromocriptine and the co-treatment were determined through western blot analysis. As shown in Fig. 3D, fisetin markedly induced high cleavage of caspase- 3 in a dose-dependent manner. Consequently, PARP was activated and apoptosis was induced. Significantly, fisetin at the highest concentration resulted in an obvious more intensive caspase-3 (Fig. 3E) and PARP (Fig. 3F) cleavage in liver cancer cells of HCC-LM3. Significantly, HCC-LM3 treated by bromocriptine at different concentrations could also stimulate caspase- 3 and PARP cleavage, which was performed in a dose-dependent manner (Fig. 3G-I). The results above show that caspase signaling pathway activation is involved in fisetin- and bromocriptine-induced apoptosis, which might be a possible molecular mechanism by which fisetin and bromocriptine exhibits strong antitumor effects.

Fisetin and bromocriptine suppresses EMT in vitro regulated by $T G F-\beta 1$. EMT is known to play an important role in cancer metastasis (21). Essentially, TGF- $\beta 1$ has been well reported to 
A
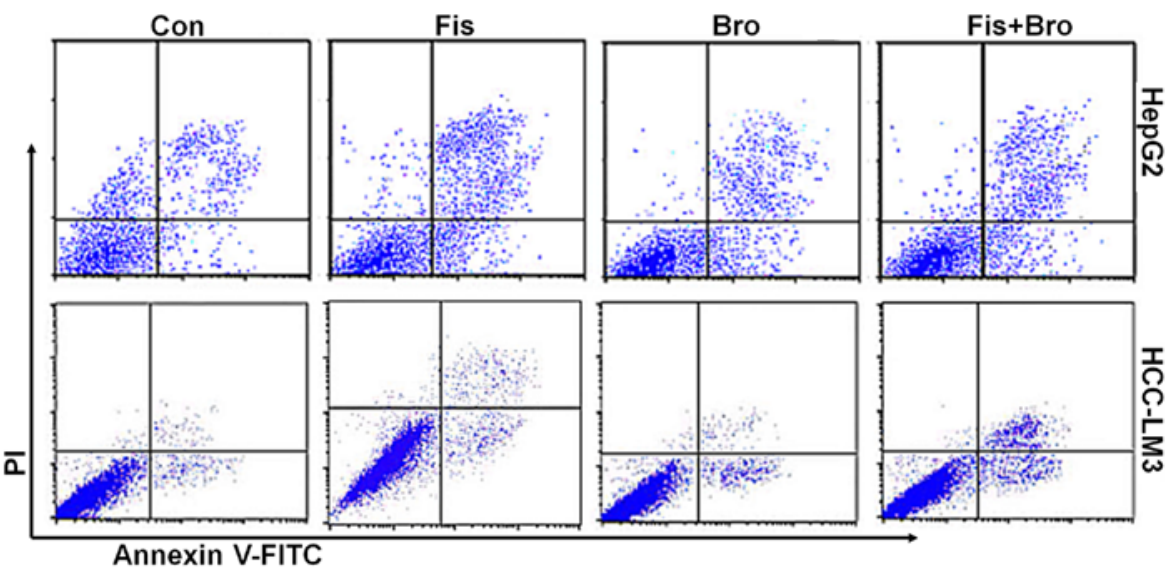

B
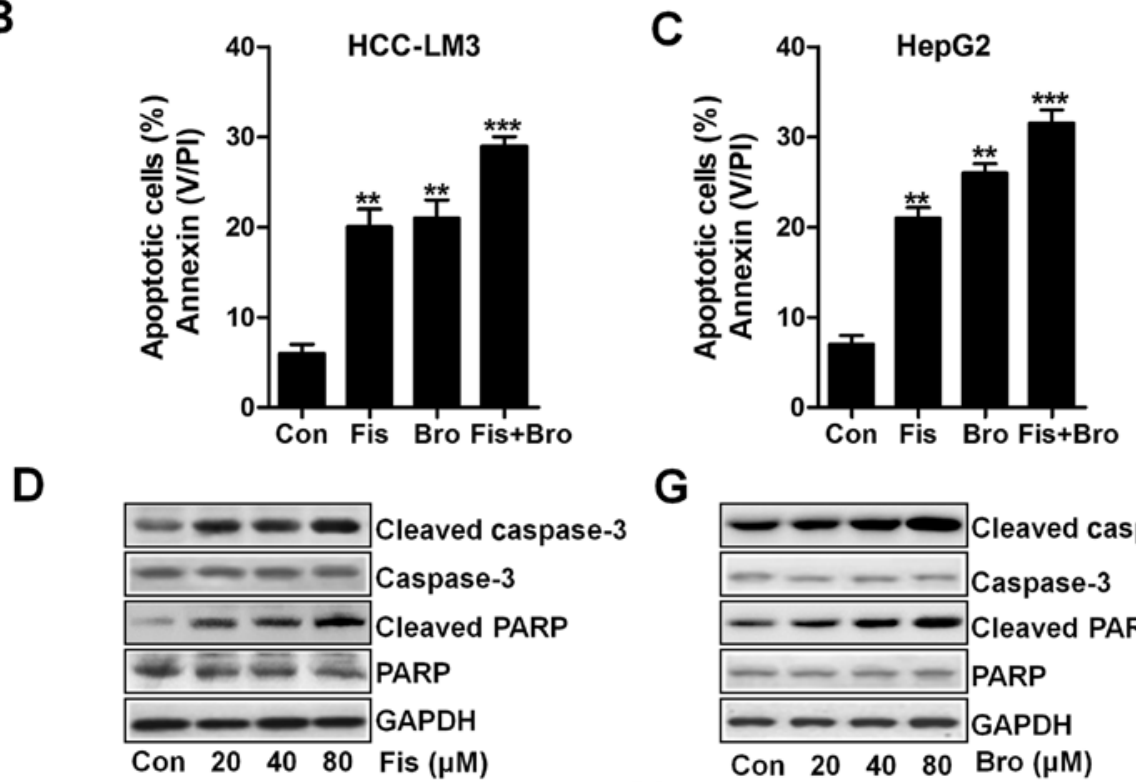

G
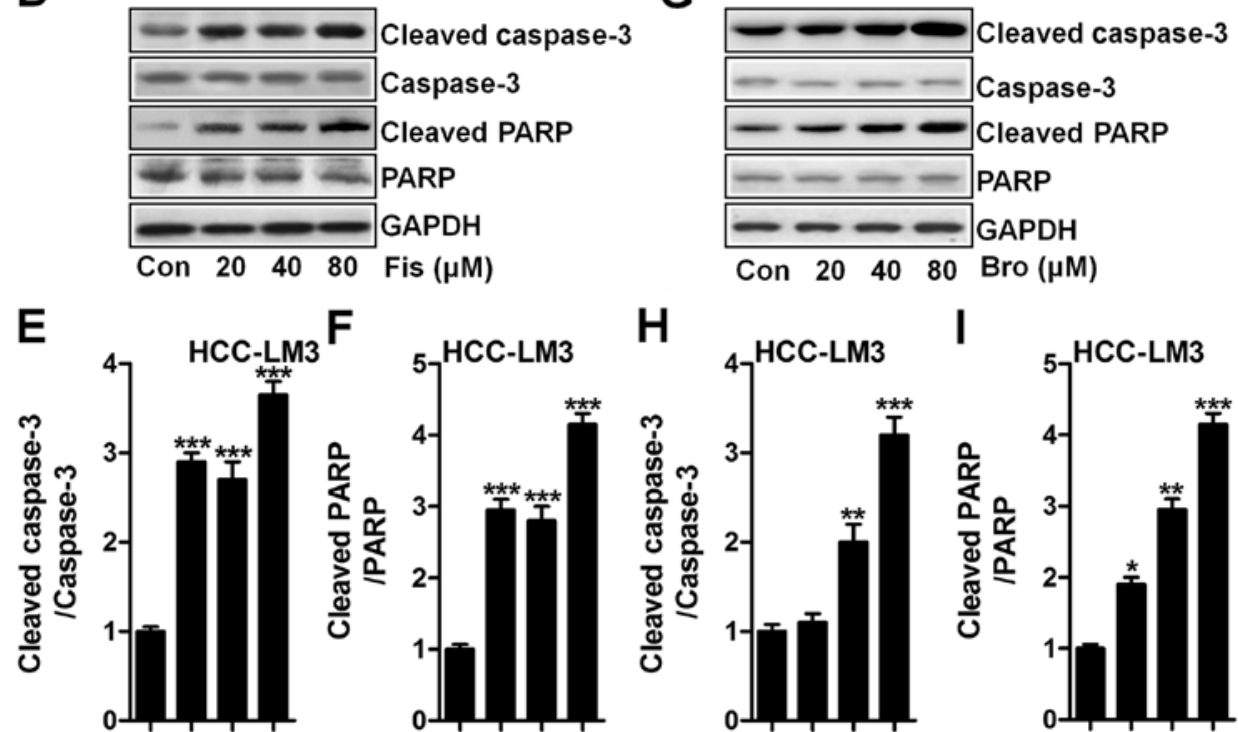

Fis $(\mu \mathrm{M})$ Con 204080 Fis $(\mu \mathrm{M})$ Con 204080
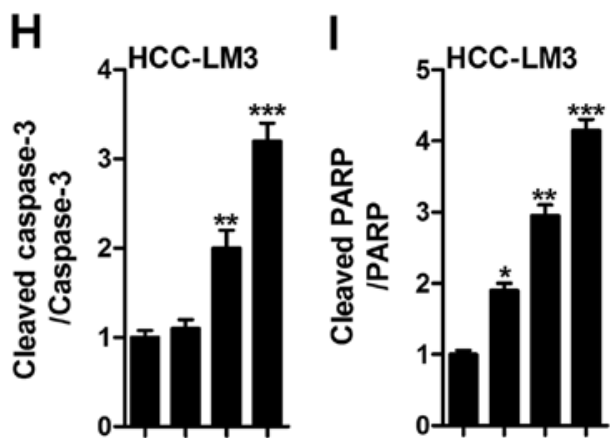

Figure 3. Fisetin and bromocriptine induce apoptosis in liver cancer cells via caspase-3 activation. (A) Apotosis of liver cancer cells was calculated via flow cytometry. (B) The quantification of HCC-LM3 experiencing apoptosis was evaluated. (C) The quantification of HepG2 in apoptosis is shown. (D) Western blot analysis was carried out to determine caspase-3 and PARP cleavage in HCC-LM3 cells after different concentrations $(0,20,40$ and $80 \mu \mathrm{M})$ of fisetin treatment. The quantification of the immunoblotting results of (E) cleaved caspase-3 and (F) cleaved PARP is shown. (G) Expression levels of caspase-3 and PARP cleavage in HCC-LM3 cells under different treatment conditions $(0,20,40$ and $80 \mu \mathrm{M})$ by bromocriptine is represented. (H) Cleaved caspase-3 and (I) cleaved PARP were quantified following western blot analysis. Data are presented as mean \pm SEM. ${ }^{*} \mathrm{P}<0.05,{ }^{* *} \mathrm{P}<0.01$ and ${ }^{* * *} \mathrm{P}<0.001$ versus Con group without any treatment.

induce the process of EMT (22). Fig. 4A shows western blot analysis of TGF- $\beta 1$ downregulated significantly compared to the control in HCC-LM3 cells after fisetin treatment, dose-dependently (Fig. 4C). In addition, vimentin (Fig. 4A and D), E-cadherin (Fig. 4A and E) and N-cadherin (Fig. 4A and $\mathrm{F}$ ) were apparently reduced due to fisetin administra- tion, which was in line with TGF- $\beta 1$ expression. Importantly, bromocriptine indicated similar role in suppressing TGF- $\beta 1$ (Fig. 4B and G), vimentin (Fig. 4B and H), E-cadherin (Fig. 4B and I) and N-cadherin (Fig. 4B and J) in HCC-LM3 cells. Furthermore, immunofluorescence analysis proved that TGF- $\beta 1$ could be reduced in fisetin and bromocriptine treat- 
A
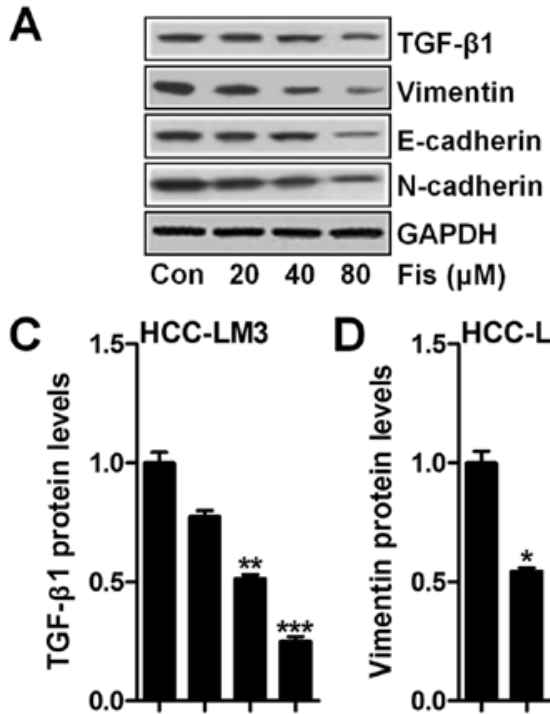

Fis $(\mu \mathrm{M})$ Con20 4080 Fis $(\mu \mathrm{M})$ Con20 4080

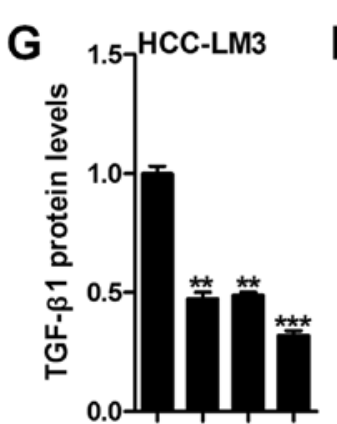

Bro ( $\mu$ M)Con 204080
D $1.5{ }^{\mathrm{HCC}-\mathrm{LM} 3} \mathrm{E}$

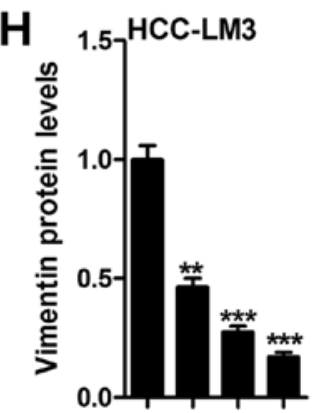

B
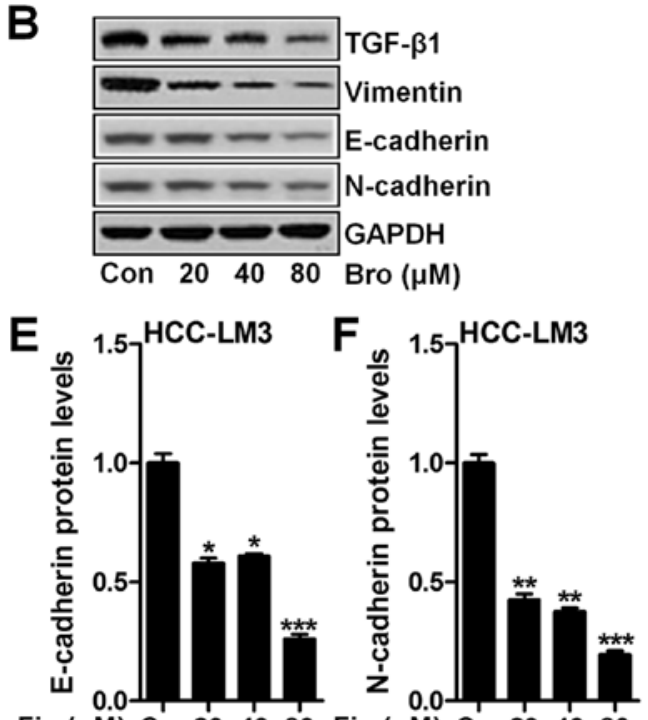

Fis $(\mu \mathrm{M})$ Con20 4080 Fis $(\mu \mathrm{M})$ Con20 4080

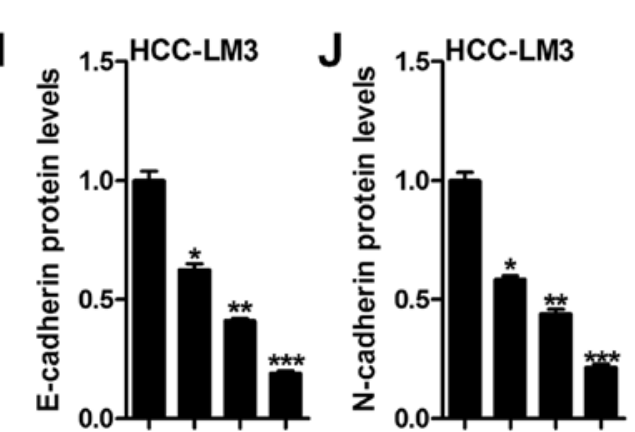

Bro $(\mu \mathrm{M}) \operatorname{Con} 204080$ Bro $(\mu \mathrm{M}) \operatorname{Con} 204080$
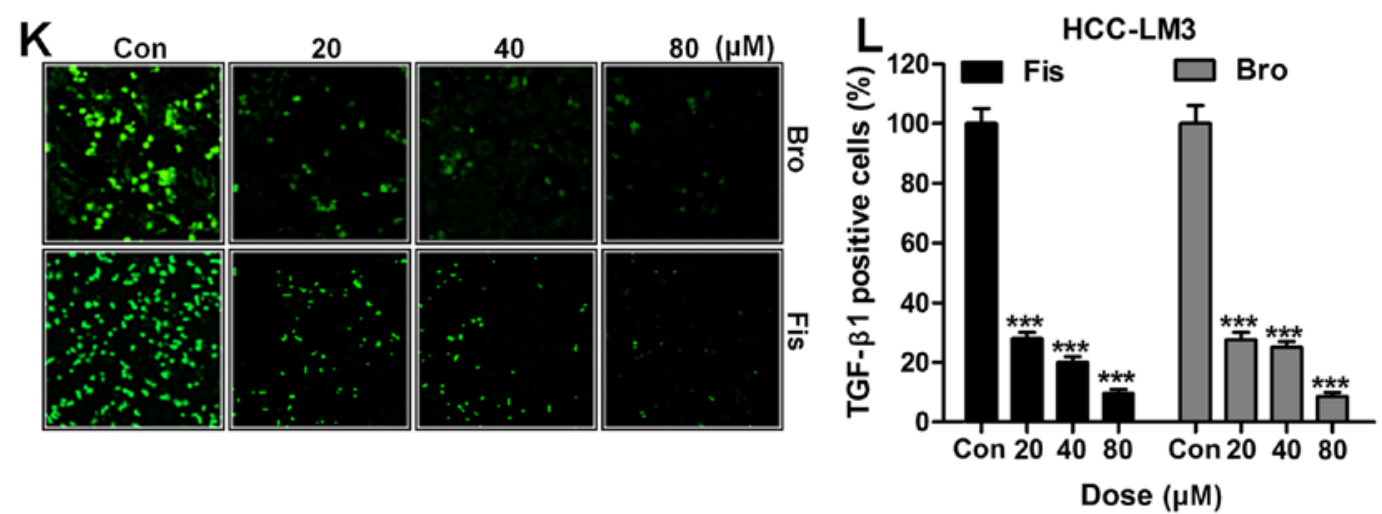

Figure 4. Fisetin and bromocriptine suppressed EMT in vitro regulated by TGF- $\beta 1$ (A) Western blot analysis of TGF- $\beta 1$, vimentin, E-cadherin and N-cadherin in HCC-LM3 cells after fisetin treatment at different concentrations. (B) Western blot analysis was used to determine TGF- $\beta 1$, vimentin, E-cadherin and $\mathrm{N}$-cadherin in HCC-LM3 cells after different concentrations of treatment by bromocriptine. The quantification of (C) TGF- $\beta 1$, (D) vimentin, (E) E-cadherin, and (F) N-cadherin was evaluated after immunoblot analysis in fisetin-treated liver cancer cells. (G) TGF- $\beta 1,(\mathrm{H})$ vimentin, (I) E-cadherin, and (J) N-cadherin protein expression levels were quantified following western blot assays in bromocriptine-treated cells. (K) The representative images of TGF- $\beta 1$ in HCC-LM3 cells after fisetin and bromocriptine treatment are shown. (L) The quantification of TGF- $\beta 1$ immunofluorescent intensity was exhibited. Data are presented as mean \pm SEM. ${ }^{*} \mathrm{P}<0.05,{ }^{* *} \mathrm{P}<0.01$ and ${ }^{* * * *} \mathrm{P}<0.001$ versus Con group without any treatment.

ment (Fig. 4K and L). The data above suggested that fisetin could ameliorate liver cancer progression via EMT inhibition through TGF- $\beta 1$ signaling pathway.

Fisetin and bromocriptine reduces the VEGFR and MAPK signaling pathways. To explore the role of fisetin and bromocriptine on VEGFR1 expression and to investigate the underlying molecular mechanism, VEGFR1, ERK1/2, p38 and pJNK expression levels were determined through western blot assays. As shown in Fig. 5A, we found that VEGFR1 (Fig. 5A and C), p-ERK1/2 (Fig. 5A and D), p38 (Fig. 5A and E) and Pjnk (Fig. 5A and F) expression levels in HCC-LM3 cells after fisetin treatment were downregulated markedly compared to the control ones. Also, in bromocriptine-treated HCC-LM3 cells, reduced protein levels of VEGFR1 (Fig. 5B and G), p-ERK1/2 (Fig. 5B and H), p38 (Fig. 5B and I) and pJNK (Fig. 5B and J) were observed. The data above indicated that fisetin could perform as bromocriptine, showing 
A

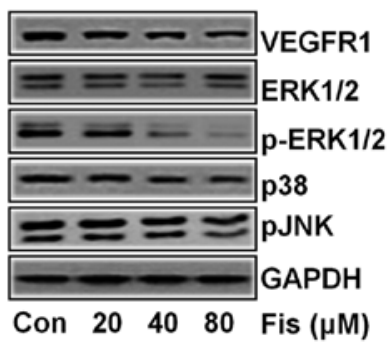

C

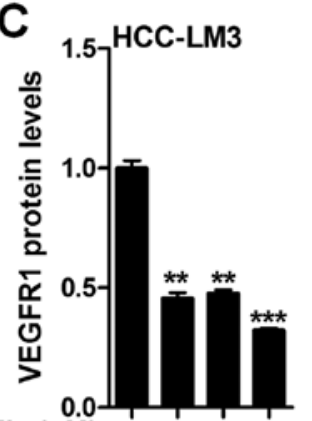

Fis $(\mu \mathrm{M})$ Con20 4080

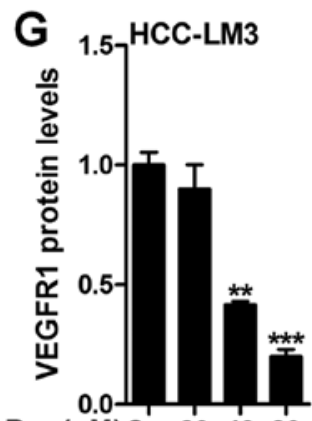

Bro $(\mu \mathrm{M})$ Con20 4080

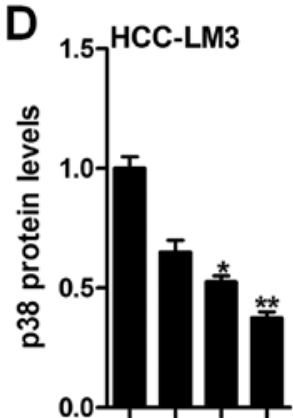

Fis $(\mu \mathrm{M})$ Con20 4080

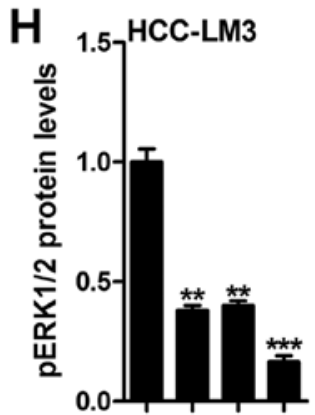

B

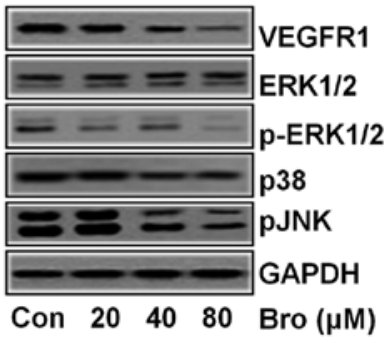

E
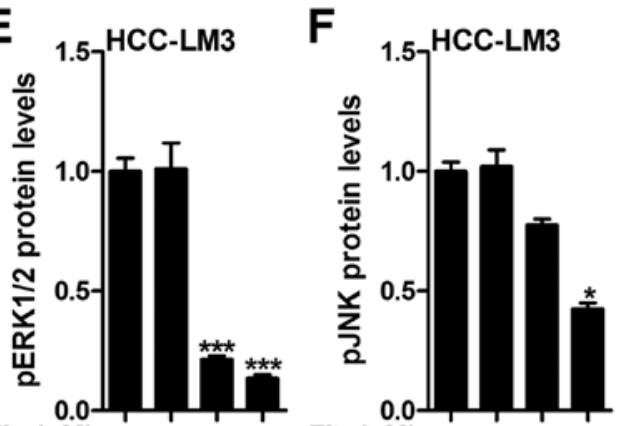

Fis $(\mu \mathrm{M})$ Con20 4080 Fis $(\mu \mathrm{M})$ Con 204080
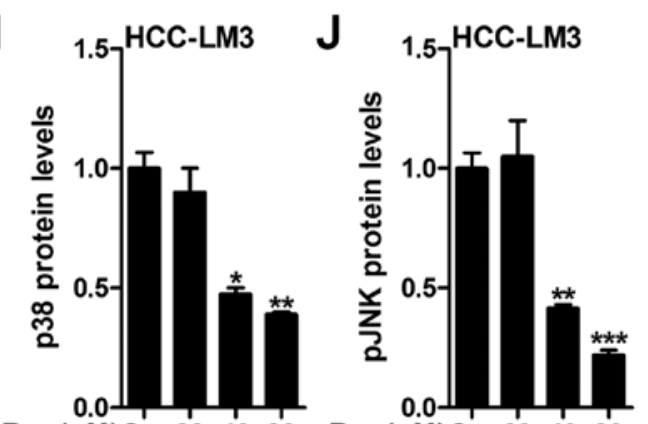

Figure 5. Fisetin and bromocriptine reduces VEGFR and MAPK signaling pathway. (A) Western blot assays of VEGFR, p-ERK1/2, ERK1/2, p38 and pJNK in HCC-LM3 cells after fisetin treatment at different concentrations. (B) Western blot assays were performed to detect protein expression levels of VEGFR, p-ERK1/2, ERK1/2, p38 and pJNK in HCC-LM3 cells after bromocriptine treatment at different concentrations. The quantification of (C) VEGFR, (D) p38, (E) p-ERK1/2, and (F) pJNK is displayed. (G) VEGFR, (H) p-ERK1/2, (I) p38, and (J) pJNK expression levels at protein level was calculated. Data are presented as mean \pm SEM. ${ }^{*} \mathrm{P}<0.05,{ }^{* *} \mathrm{P}<0.01$ and ${ }^{* * * *} \mathrm{P}<0.001$ versus Con group without any treatment.

suppressive role in liver cancer progression via VEGFR1 and EMT-related signaling pathway inhibition.

Fisetin inhibits liver cancer growth of transplanted liver cancer cell line. In this regard, the role of fisetin i.p. injection in vivo study was evaluated. As shown in Fig. 6A and $\mathrm{B}$, fisetin administration significantly reduced the weight of tumors, isolated from mice with orthotopically implanted tumors. Also, survival analysis showed that fisetin treatment significantly prolonged the survival time of mice with orthotopically implanted tumors in comparison to the control ones (Fig. 6C). Finally, DA levels were measured via ELISA kits. In the serum (Fig. 6D) and tumor tissue (Fig. 6E) from mice with orthotopically implanted tumors, DA was expressed highly, which was downregulated significantly after fisetin treatments in a dose-dependent manner.

Next, immunohistochemical analysis was used to detect Ki-67, and VEGFR1 levels from tumor specimens. As shown in Fig. 7A and B, Ki-67 was significantly downregulated due to fisetin administration, which was dose-dependent. Similarly, VEGFR1 was also impeded in fisetin-treated group, which was in line with the results in vitro (Fig. 7C and D). TGF- $\beta 1$ was reduced in tumor tissue samples isolated from fisetin-treated mice through immunofluorescent analysis (Fig. 7E and F). The data above indicated that fisetin could suppress liver cancer development, which was related to death receptor signaling.

\section{Discussion}

Hepatocellular carcinoma (HCC) is reported as the most common form of primary liver cancer. There is still no effective therapies to prevent it $(1-3,23)$. Compounds with bioactivity extracted from various plants are catching attention due to their less toxicity and more effective activity in controlling disease progression (24). For instance, the isolated and purified fatty acid from A. spinosus was discovered to inhibit the HepG2 cell proliferation (25). In our study, similar role was found by the use of fisetin. Fisetin changed DA levels in the serum of mice as well as in the tumor tissue samples. In addition, fisetin performs as DR2 agonist in regulating liver cancer cell proliferation, suggesting that on the one hand, the nervous system is involved in HCC development. Also, fisetin might be used as a crucial and effective agent in HCC suppression. 
A

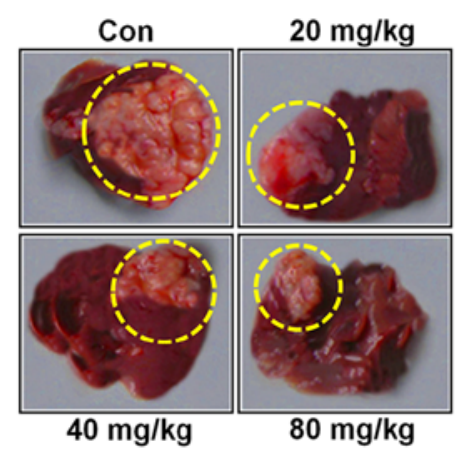

C

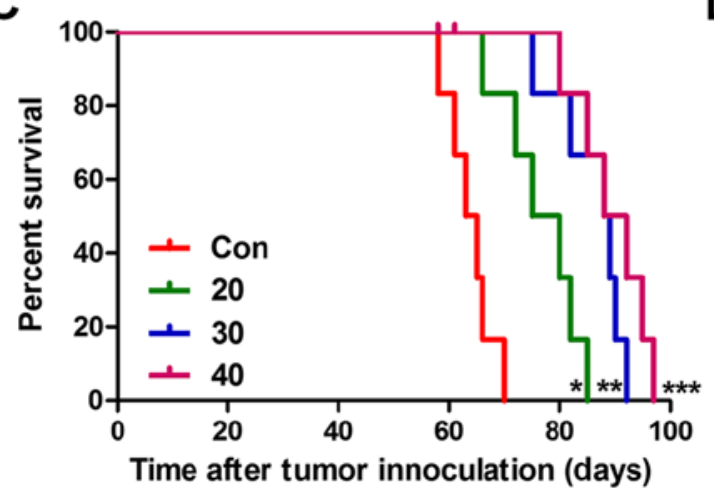

B

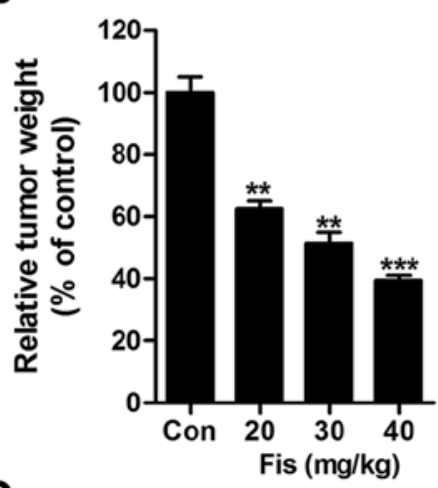

D

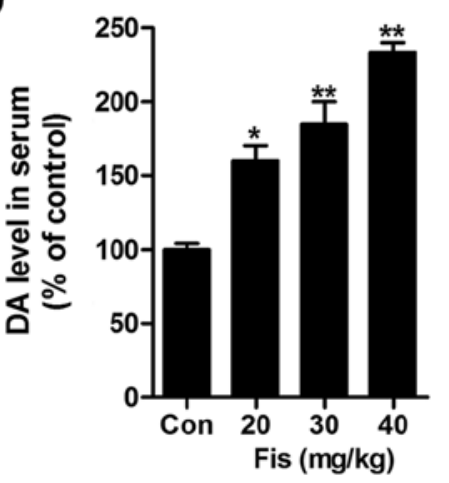

$\mathbf{E}$

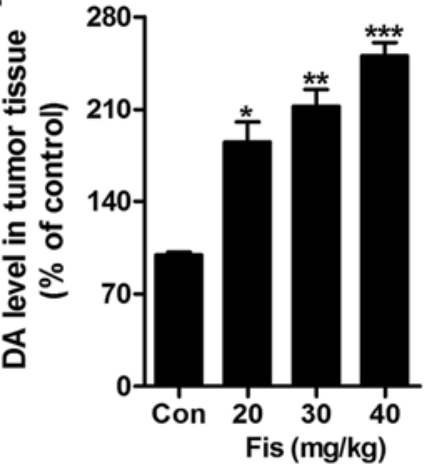

Figure 6. Fisetin inhibits liver cancer growth of transplanted liver cancer cell line (A) Representative images of tumors from BALB/C nude mice, which were orthotopically implanted with the HCC-LM3-RFP cells. Immediately after implantation, the animals were administered with different concentrations ( 0,20 , 30 and $40 \mathrm{mg} / \mathrm{kg}$ ) of fisetin daily via i.p. injection. After 7 weeks of treatment, the mice were sacrificed for following experiments. (B) The weight of tumor tissue from mice was measured. (C) The percent of survival rate was shown in mice with orthotopically implanted tumors in the absence or presence of fisetin. DA levels (D) in serum and (E) tumor tissue samples from mice with orthotopically implanted tumors were calculated via ELISA kits. Data are represented as mean \pm SEM. ${ }^{*} \mathrm{P}<0.05,{ }^{* *} \mathrm{P}<0.01$ and ${ }^{* * * *} \mathrm{P}<0.001$ versus Con group without any treatment.

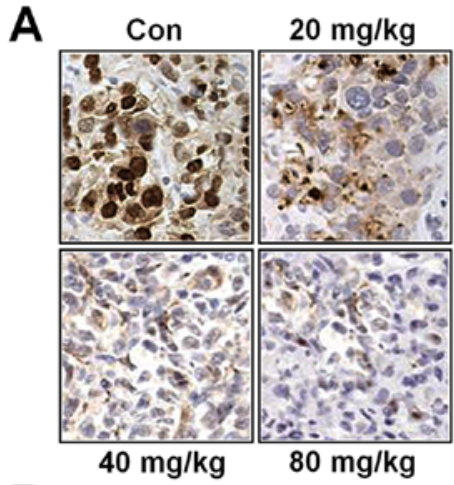

B

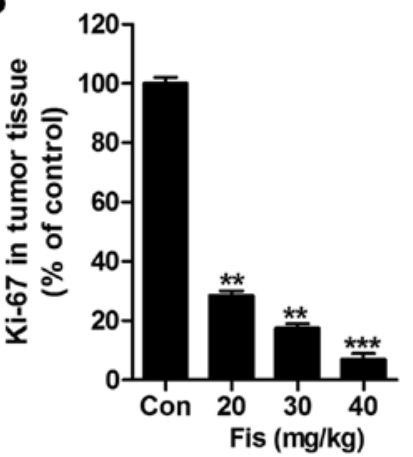

C
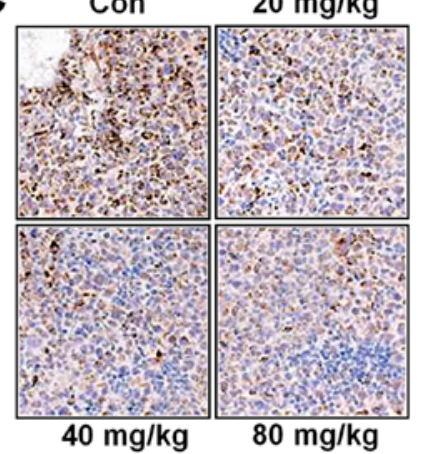

D

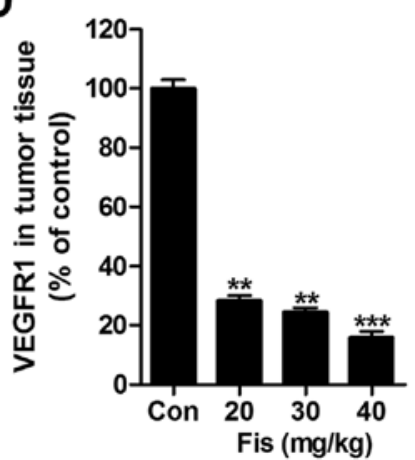

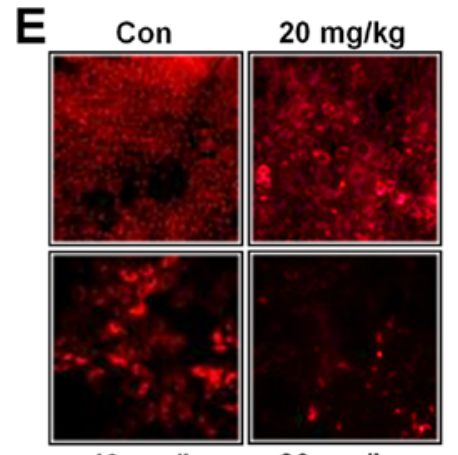

$\mathbf{F}$

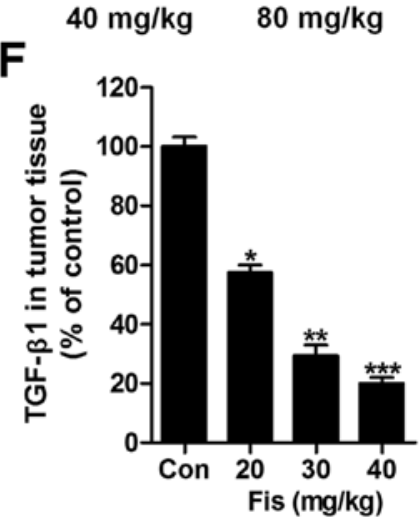

Figure 7. Fisetin treatment amelioarates liver cancer progression related to VEGFR 1 and TGF- $\beta 1$ regulation (A) Immunohistochemical (IHC) analysis of $\mathrm{Ki}$-67 is shown. (B) Ki-67 expressed levels via IHC were quantified. (C) VEGFR1 expression levels were determined via IHC. (D) VEGFR1-positive cells were calculated in the tumor tissue samples from mice. (E) Immunofluorescent (IF) analysis was used to determine TGF- $\beta 1$ levels in tumor tissue samples from mice with or without fisetin treatment at different concentrations. (F) The quantification of TGF- $\beta 1$ is shown accroding to the IF results. Data are represented as mean \pm SEM. ${ }^{*} \mathrm{P}<0.05,{ }^{* *} \mathrm{P}<0.01$ and ${ }^{* * * *} \mathrm{P}<0.001$ versus Con group without any treatment. 
First in this study, fisetin was found to inhibit liver cancer cell proliferation, migration and invasion. It has been suggested that apoptosis induction is a main molecular mechanism by which cancer cells experience cell death (26). Also, presently, many drugs used for apoptosis induction are explored in order to find effective therapeutic strategies for cancer prevention (27). The caspase-related mechanism is considered as a major signal pathway resulting in apoptosis (28). Disorders of caspases are related to various cancer cell immortality (29). In our study, we found that fisetin could activate caspase- 3 activity. The cleaved caspase- 3 was expressed highly with the increasing of fisetin treatment, which was in line with the effects of bromocriptine on HCC-LM3 cells, suggesting that fisetin, to some degree, shows similar role with bromocriptine in regulating liver cancer. Subsequently, PARP cleavage was improved, activating apoptosis eventually. Also, our flow cytometry analysis indicated that apoptosis was induced for fisetin, bromocriptine and the two combinations. Of note, fisetin and bromocriptine co-treatment could further upregulate apoptosis, which might be due to fisetin administration enhancing bromocriptine role in liver cancer apoptosis induction and promoting cell death.

Previous studies have suggested that dopamine has close relationship with stress and movement (30). As a significant part of the nervous system, dopamine plays important role in development of many diseases, including cancer (31). Dopamine has been indicated to possess anticancer properties (32). In our study, we found that dopamine was upregulated in the serum and tumor tissue samples of mice with liver cancer for fisetin administration. The data indicated that dopamine was also involved in liver cancer progression, and fisetin could suppress liver cancer development, which was related to dopamine mediation. However, further study is needed to investigate the specific relationship between fisetin and dopamine in liver cancer progression. TGF- $\beta 1$ is well known in tumor growth, which could be regulated for ERK signaling pathway (33). ERK signaling pathway could activate TGF- $\beta 1$ expression, contributing to various tumor metastasis (34). In our study, TGF- $\beta 1$ was highly expressed in liver cancer cells and tumors, which was downregulated for fisetin administration, as well as bromocriptine treatment. Also, the following signals of p38 and pJNK were inhibited for fisetin and bromocriptine, accompanied with vimentin, E-cadherin and $\mathrm{N}$-cadherin, which are important members associated with tumor growth $(35,36)$.

In conclusion, this study indicated that fisetin could influence the nervous system, at least partly, as dopamine receptors function. Fisetin shows suppressive role in liver cancer development, which could reduce the volume of liver cancer and prolong the survival of mice. Fisetin could be considered as dopamine agonist to inhibit liver cancer progression. Our study indicated that fisetin was beneficial for liver cancer inhibition. However, further study is still needed to clarify the molecular mechanism by which fisetin influences dopamine in the nervous system.

\section{References}

1. El-Serag HB and Rudolph KL: Hepatocellular carcinoma: Epidemiology and molecular carcinogenesis. Gastroenterology 132: 2557-2576, 2007.

2. Rampone B, Schiavone B, Martino A, Viviano C and Confuorto G: Current management strategy of hepatocellular carcinoma. World J Gastroenterol 15: 3210-3216, 2009.
3. Rahbari NN, Mehrabi A, Mollberg NM, Müller SA, Koch M, Büchler MW and Weitz J: Hepatocellular carcinoma: Current management and perspectives for the future. Ann Surg 253: 453-469, 2011.

4. Xu G, Qi FZ, Zhang JH, Cheng GF, Cai Y and Miao Y: Metaanalysis of surgical resection and radiofrequency ablation for early hepatocellular carcinoma. World J Surg Oncol 10: 163, 2012.

5. DuBray BJ Jr, Chapman WC and Anderson CD: Hepatocellular carcinoma: A review of the surgical approaches to management. Mo Med 108: 195-198, 2011.

6. Salhab M and Canelo R: An overview of evidence-based management of hepatocellular carcinoma: A meta-analysis. J Cancer Res Ther 7: 463-475, 2011.

7. De Minicis S,Candelaresi C,Marzioni M,Saccomano S, Roskams T, Casini A, Risaliti A, Salzano R, Cautero N, di Francesco F, et al: Role of endogenous opioids in modulating HSC activity in vitro and liver fibrosis in vivo. Gut 57: 352-364, 2008.

8. Peinado H, Alečković M, Lavotshkin S, Matei I, Costa-Silva B, Moreno-Bueno G, Hergueta-Redondo M, Williams C, GarcíaSantos G, Ghajar C, et al: Melanoma exosomes educate bone marrow progenitor cells toward a pro-metastatic phenotype through MET. Nat Med 18: 883-891, 2012.

9. Kohno H, Sakai T, Saito S, Okano K and Kitahara K: Treatment of experimental autoimmune uveoretinitis with atorvastatin and lovastatin. Exp Eye Res 84: 569-576, 2007.

10. Byun MW: Schizonepeta tenuifolia ethanol extract exerts antiinflammatory activity through the inhibition of TLR4 signaling in lipopolysaccharide-stimulated macrophage cells. J Med Food 17: 350-356, 2014.

11. Li J, Cheng Y, Qu W, Sun Y, Wang Z, Wang H and Tian B: Fisetin, a dietary flavonoid, induces cell cycle arrest and apoptosis through activation of p53 and inhibition of NF-kappa B pathways in bladder cancer cells. Basic Clin Pharmacol Toxicol 108: 84-93, 2011.

12. Ying TH, Yang SF, Tsai SJ, Hsieh SC, Huang YC, Bau DT and Hsieh YH: Fisetin induces apoptosis in human cervical cancer HeLa cells through ERK1/2-mediated activation of caspase-8-/ caspase-3-dependent pathway. Arch Toxicol 86: 263-273, 2012.

13. Contreras F, Fouillioux C, Bolívar A, Simonovis N, HernándezHernández R, Armas-Hernandez MJ and Velasco M: Dopamine, hypertension and obesity. J Hum Hypertens 16 (Suppl 1): S13-S17, 2002.

14. Hazelwood LA, Free RB, Cabrera DM, Skinbjerg M and Sibley DR: Reciprocal modulation of function between the D1 and $\mathrm{D} 2$ dopamine receptors and the $\mathrm{Na}^{+}, \mathrm{K}^{+}$-ATPase. $\mathrm{J}$ Biol Chem 283: 36441-36453, 2008.

15. Salyer S, Lesousky N, Weinman EJ, Clark BJ, Lederer ED and Khundmiri SJ: Dopamine regulation of $\mathrm{Na}^{+}-\mathrm{K}^{+}$-ATPase requires the PDZ-2 domain of sodium hydrogen regulatory factor-1 (NHERF-1) in opossum kidney cells. Am J Physiol Cell Physiol 300: C425-C434, 2011.

16. Zhang YR and Yuan ZY: Dopamine-mediated inhibition of renal $\mathrm{Na}^{+} / \mathrm{K}^{+}$-ATPase in HK-2 cells is reduced by ouabain. Clin Exp Pharmacol Physiol 37: 613-618, 2010.

17. Calarge CA, Ellingrod VL, Acion L, Miller DD, Moline J, Tansey MJ and Schlechte JA: Variants of the dopamine D2 receptor gene and risperidone-induced hyperprolactinemia in children and adolescents. Pharmacogenet Genomics 19: 373-382, 2009.

18. Trussardi-Regnier A, Lavenus S, Gorisse MC and Dufer J: Thalidomide alters nuclear architecture without $\mathrm{ABCB} 1$ gene modulation in drug-resistant myeloma cells. Int J Oncol 35: 641-647, 2009.

19. Cagin YF, Parlakpinar H, Vardi N, Polat A, Atayan Y, Erdogan MA and Tanbek K: Effects of dexpanthenol on acetic acid-induced colitis in rats. Exp Ther Med 12: 2958-2964, 2016.

20. Yang PM, Tseng HH, Peng CW, Chen WS and Chiu SJ: Dietary flavonoid fisetin targets caspase-3-deficient human breast cancer MCF-7 cells by induction of caspase-7-associated apoptosis and inhibition of autophagy. Int J Oncol 40: 469-478, 2012.

21. Christiansen JJ and Rajasekaran AK: Reassessing epithelial to mesenchymal transition as a prerequisite for carcinoma invasion and metastasis. Cancer Res 66: 8319-8326, 2006.

22. Miyazono K, Ehata S and Koinuma D: Tumor-promoting functions of transforming growth factor- $\beta$ in progression of cancer. Ups J Med Sci 117: 143-152, 2012.

23. Wheelhouse NM, Lai PB, Wigmore SJ, Ross JA and Harrison DJ: Mitochondrial D-loop mutations and deletion profiles of cancerous and noncancerous liver tissue in hepatitis B virusinfected liver. Br J Cancer 92: 1268-1272, 2005.

24. van Zijl F, Zulehner G, Petz M, Schneller D, Kornauth C, Hau M, Machat G, Grubinger M, Huber H and Mikulits W: Epithelialmesenchymal transition in hepatocellular carcinoma. Future Oncol 5: 1169-1179, 2009 
25. Jin Z, McDonald ER III, Dicker DT and El-Deiry WS: Deficient tumor necrosis factor-related apoptosis-inducing ligand (TRAIL) death receptor transport to the cell surface in human colon cancer cells selected for resistance to TRAIL-induced apoptosis. J Biol Chem 279: 35829-35839, 2004.

26. Kim HS, Lee JW, Soung YH, Park WS, Kim SY, Lee JH, Park JY, Cho YG, Kim CJ, Jeong SW, et al: Inactivating mutations of caspase- 8 gene in colorectal carcinomas. Gastroenterology 125 : $708-715,2003$

27. Zhang S, Ong $\mathrm{CN}$ and Shen HM: Involvement of proapoptotic Bcl-2 family members in parthenolide-induced mitochondrial dysfunction and apoptosis. Cancer Lett 211: 175-188, 2004.

28. Pennarun B, Meijer A, de Vries EG, Kleibeuker JH, Kruyt F and de Jong S: Playing the DISC: Turning on TRAIL death receptormediated apoptosis in cancer. Biochim Biophys Acta 1805. 123-140, 2010.

29. Jung YH, Heo J, Lee YJ, Kwon TK and Kim YH: Quercetin enhances TRAIL-induced apoptosis in prostate cancer cells via increased protein stability of death receptor 5. Life Sci 86: 351-357, 2010.

30. Jia L and Zhang MH: Comparison of probiotics and lactulose in the treatment of minimal hepatic encephalopathy in rats. World J Gastroenterol 11: 908-911, 2005.
31. Yamada M, Chiba T, Sasabe J, Nawa M, Tajima H, Niikura T, Terashita K, Aiso S, Kita Y, Matsuoka M, et al: Implanted cannula-mediated repetitive administration of Abeta25-35 into the mouse cerebral ventricle effectively impairs spatial working memory. Behav Brain Res 164: 139-146, 2005.

32. Seamans JK and Yang CR: The principal features and mechanisms of dopamine modulation in the prefrontal cortex. Prog Neurobiol 74: 1-58, 2004

33. Lu Q: Transforming growth factor-betal protects against pulmonary artery endothelial cell apoptosis via ALK5. Am J Physiol Lung Cell Mol Physiol 295: L123-L133, 2008.

34. Baarsma HA, Spanjer AI, Haitsma G, Engelbertink LH, Meurs H, Jonker MR, Timens W, Postma DS, Kerstjens HA and Gosens R: Activation of WNT/ $\beta$-catenin signaling in pulmonary fibroblasts by TGF- $\beta_{1}$ is increased in chronic obstructive pulmonary disease. PLoS One 6: e25450, 2011.

35. Liu GL, Yang HJ, Liu T and Lin YZ: Expression and significance of E-cadherin, $\mathrm{N}$-cadherin, transforming growth factor- $\beta 1$ and Twist in prostate cancer. Asian Pac J Trop Med 7: 76-82, 2014.

36. Lee JM, Dedhar S, Kalluri R and Thompson EW: The epithelialmesenchymal transition: New insights in signaling, development, and disease. J Cell Biol 172: 973-981, 2006. 